\title{
Influencia del aprendizaje organizacional en la calidad de la educación de una IES
}

\author{
Influence of organizational learning on the quality of education in an HEI
}

\author{
Paulo García-Bravo \\ Escuela de Formación Infantería de Marina - Colombia \\ ORCID iD: https://orcid.org/0000-0001-9044-9714 \\ paulogarcial@armada.mil.co
}

\author{
Fecha de recepción: 12/08/2020 \\ Fecha de evaluación: 11/10/2020 \\ Fecha de aceptación: 29/10/2020
}

Cómo citar: García-Bravo, P. (2020). Influencia del aprendizaje organizacional en la calidad de la educación de unaIES. Revista CientíficaAnfibios, 3(2), 11-21. https://doi.org/10.37979/afb.2020v3n2.68

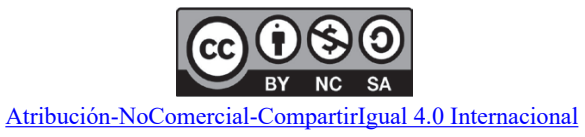

\section{Resumen}

En las organizaciones, y en especial en las militares, se requiere que los conocimientos acerca de los procesos y tareas se puedan mejorar con la finalidad de corresponder ante los retos y desafíos que propone el mundo globalizado actual. Es así como se propone estudiar la influencia del aprendizaje organizacional en la calidad educativa en una IES, de forma concreta en la EFIM, a través de modelos de ecuaciones estructurales que permitan establecer la asociación entre estos constructos y su grado de influencia. Participaron 64 Directivos y Docentes de una Escuela de Formación Militar de la Armada Nacional de Colombia. El diseño metodológico se abordó a través del Análisis de Conglomerados Jerárquicos, Análisis factorial exploratorio y Método de Ecuaciones Estructurales. Como resultados de planteo un análisis de las correlaciones significativas entre calidad educativa y aprendizaje organizacional, determinando que existe una asociación significativa entre los dos constructos.

Palabras clave:

Aprendizaje organizacional; calidad educativa; castrense; incidencia; conglomerados.

\begin{abstract}
In organizations, and especially in the Army, knowledge about processes and tasks needs to be improved in order to respond to the challenges of today's globalized world. Thus, it is proposed to study the influence of organizational learning on the educational quality in an HEI, specifically in the EFIM, through structural equation models that allow establishing the association between these constructs and their degree of influence. Sixty-four directors and teachers of a Military Training School of the Colombian Navy participated. The methodological design was approached through the Hierarchical Cluster Analysis, Exploratory Factor Analysis and Structural Equations Method. As a result, I propose an analysis of the significant correlations between educational quality and organizational learning, determining that there is a significant association between the two constructs.
\end{abstract}


Keywords:

Organizational learning; quality education; military; advocacy; clusters.

\section{Introducción}

El aprendizaje organizacional (AO) en la calidad educativa es una temática relativamente nueva en el campo de la investigación académica, evidenciándose pocos antecedentes que muestren relación entre estas dos variables. Sin embargo, en la revisión de estudios anteriores se presentaron investigaciones relacionadas con la temática abordada desde una perspectiva teórico-metodológica, identificando que el aprendizaje organizacional en el ámbito de la educación ha sido poco estudiado, puesto que es una concepción originada en el campo empresarial y trasladada al ámbito educativo.

Diversas investigaciones han relacionado la calidad educativa, vista desde el rendimiento académico de los alumnos, con múltiples conceptos que abarcan diferentes variables, como la educación de los padres (Barrientos, 2001), número de docentes por alumno (Barrientos, 2001), filosofía organizacional y procesos administrativos (Ramírez-Fernández, Machado-Licona \& Fernández-Ramírez), características del aula y flexibilidad curricular (Schiefelbein \& Schiefelbein, 1999), determinantes personales (Garbanzo, 2014), entre otros, no obstante, su relación con el factor de aprendizaje organizacional, ha sido poco relacionado aún en la literatura, presentando una oportunidad de investigación viéndolo desde la óptica de López \& López (2003), quien describe el aprendizaje organizativo como el proceso mediante el cual se integran conocimientos, habilidades y actitudes para conseguir cambios y mejoras en la organización.

Es así como se dispuso realizar una investigación que permitiera analizar cuál es la influencia que tiene el aprendizaje organizacional en la calidad de la educación que se imparte en las Instituciones de Educación Superior (IES en adelante), con especial énfasis en el apartado militar y tomando como referencia específica la Escuela de Formación de Infantería de Marina, en adelante conocida como la EFIM, entendiendo que es la institución responsable de formar militar y profesionalmente los alum- nos como Suboficiales de Infantería de Marina desde 1956, año en el cual fue fundada. Desde entonces y como institución militar, los estudiantes son instruidos en un contexto académico militar, por docentes militares y civiles durante 24 meses, siendo mayor el porcentaje de profesores militares por la misma naturaleza del ente educativo.

Por su naturaleza, la cultura organizacional en las instituciones castrenses está arraigada por la doctrina militar, que tiene su fundamento en el conductismo, teoría que ha sido estudiada inicialmente en los Estados Unidos en el siglo XX por el Psicólogo John Watson, pero también otros importantes estudiosos como Iván Pávlov, Edward Thorndike, Burrhus Frederic Skinner, Albert Bandura, entre otros, realizaron valiosos aportes a este estilo de aprendizaje (García-Bravo et al., 2019). La fórmula conductista de Watson expresa que es la reacción a un estímulo, cuya fórmula es $R=f(E)$, donde $E$ es estímulo, $\mathrm{R}$ es reacción y f significa función de, es decir, los elementos de la conducta son dos fenómenos físicos: el estímulo y la reacción, siendo la conducta la conexión entre ambos y la reacción es la función del estímulo (Mariano, 1996).

El liderazgo es también un aspecto relevante en la formación de los estudiantes de la EFIM, por cuanto los alumnos una vez egresan con el grado de Cabo Tercero de Infantería de Marina, inician el proceso productivo en el nivel de Suboficial, debiendo comandar y dirigir Infantes de Marina Profesionales, Regulares o Bachilleres en diferentes escenarios de la geografía colombiana, donde el liderazgo no debe ser una exigencia institucional sino una necesidad personal y profesional, para poder comandar la tropa que le sea asignada, fundada bajo el ejemplo permanente. Moreira (2010) citando a Syroit, define de una forma simple el término de liderazgo, como: "un conjunto de actividades de un individuo que ocupa una posición jerárquicamente superior, dirigida a la realización y dirección de las actividades de otros miembros con el objeto de lograr de manera efectiva el objetivo del grupo". 
En este contexto, la EFIM como IES, tiene la responsabilidad de garantizar la calidad de los Suboficiales que semestralmente egresan desde sus claustros hacia diferentes lugares del país, tanto en la preparación militar como en la formación académica; pero existen diferentes variables que limitan la calidad del producto esperado, como es el caso de aquellos jóvenes que deciden enlistarse en instituciones de nivel tecnológico militar, quienes generalmente poco les interesa capacitarse, pues la premisa en la mayoría de los casos, es poder devengar un sueldo lo antes posible, considerando las necesidades personales y familiares, situación que genera porcentajes considerables en promedios académicos inferiores al requerido por la escuela y un índice de deserción estudiantil especialmente en el primer semestre.

En este sentido y conscientes de que la calidad educativa es un deber dentro de las Escuelas de Formación de la Armada Nacional, la EFIM ha venido interpretando que el aprendizaje organizacional juega un papel relevante al interior de la institución, entendiendo este concepto como el proceso de crear, retener y transferir el conocimiento dentro de la organización, el cual mejora con el tiempo a medida que se adquiere experiencia y esta experiencia, capaz de crear conocimiento, refiere que el aprendizaje organizacional ocurre como una función de la experiencia dentro de la organización, pues el hecho de que el ente está constantemente construyendo y reconstruyendo la realidad en la cual está inserta, sería lo que daría lugar a nuevos aprendizajes (Figueroa, 2002) y por ello esta investigación tuvo como objetivo determinar la influencia del aprendizaje organizacional en la calidad educativa de la EFIM, a través de modelos de ecuaciones estructurales que permita establecer la asociación entre estos constructos y su grado de influencia.

Para lograr el propósito planteado, la calidad educativa se analizó desde la evaluación realizada por los estudiantes de la institución durante el periodo 2018-I, donde se estudió la relación del aprendizaje organizacional desde los docentes en la calidad educativa percibida por los alumnos, por cuanto una enseñanza de calidad en educación superior debe cambiar la manera como los estudiantes comprenden, ex- perimentan o conceptualizan el mundo que les rodea (Sánchez, R. \& Jiménez, K. - 2017).

Es así como se ha entendido que el aprendizaje organizacional está basado en el aprendizaje de sus trabajadores, puesto que son las personas las que aprenden y construyen conocimientos con otros, gracias a ese proceso colectivo de transferencia de conocimientos, pues una institución que aprende a través de sus colaboradores, facilita el flujo de conocimiento de nivel individual al grupal y al organizacional y viceversa. En otras palabras, (Medina, 2017) citando a Castaneda \& Fernández (2007), menciona que la base del aprendizaje organizacional es el aprendizaje individual, a través de la cual una entidad adquiere o crea conocimiento con el propósito de adaptarse a las condiciones de su entorno o transformándolo, según sea su nivel de desarrollo.

Para el desarrollo de estos objetivos, se inicia con una aproximación teórica en donde se plantea el aprendizaje organizacional y la calidad educativa con sus respectivas dimensiones, posteriormente se plantea el desarrollo metodológico que incluye análisis de conglomerados y el planteamiento de ecuaciones estructurales para el modelo. Seguidamente se plantean los hallazgos en donde analizan los conglomerados tomando en cuenta las dimensiones anteriormente mencionadas y finalmente se esbozan algunas conclusiones y discusiones.

\section{Aproximación Teórica}

\section{Aprendizaje Organizacional}

Para Del Río Cortina \& Santisteban, (2011) el AO es un proceso en el cual las entidades, grandes o pequeñas, públicas o privadas, adquieren y crean conocimiento a través de sus empleados, con el objetivo de transformarlo en conocimiento institucional para que la organización pueda adaptarse a las circunstancias cambiantes o transformarlas. En ese orden de ideas, las organizaciones que desarrollan capacidades de aprendizaje organizacional comienzan con procedimientos simples de rutina que no cuestionan la estructura de la organización, su relación con el entorno, su valor o el proceso de toma de decisiones. Luego, en el segundo nivel que 
persigue el AO, inicia con el aprendizaje personal de los colaboradores y cuestionando la racionalidad detrás de la acción y procesos en los aspectos mencionados (Argyris \& Schon, 1999).

Teniendo en cuenta que AO depende de los procesos necesarios para adquirir, difundir y utilizar el conocimiento, este resulta como una "memoria organizativo" de la institución (Bolívar, 2001). Para Garvin et al., (2008) el AO depende de tres componentes; el primero hace referencia al ambiente de soporte de aprendizaje, el cual vincula aspectos como la seguridad psicológica, la cual se refleja en que cualquier empleado puede preguntar cuestionamientos acerca de sus tareas a sus superiores, la apreciación de las diferencias en el cual surge un conocimiento entre ideas contrarias y la disposición del empleado para aprender o su apertura a nuevas ideas y el tiempo para reflexionar, que equivale al lapso donde el empleado es consciente de sus tareas y a partir de aquí comienza a racionalizar y entender su papel dentro de la organización.

El segundo componente se vincula con procesos y prácticas del aprendizaje, en el cual el conocimiento debe ser compartido de manera sistemática y claramente definida. El intercambio puede tener lugar entre individuos, grupos $\mathrm{u}$ organizaciones enteras vinculando procesos de generación, recolección, interpretación y difusión de información.

El tercer componente se trata del liderazgo y el refuerzo del aprendizaje, el cual los líderes de la organización señalan lo importante que es dedicar tiempo a la identificación de problemas, la transferencia de conocimientos y la revisión de seguimiento reflexiva fomentando el conocimiento y su transferencia en la institución. Cuando el líder demuestra su disposición a informarse respecto a otros puntos de vista a través de sus acciones, el empleado se siente audaz y presenta nuevas ideas y opciones promoviendo el conocimiento al interior de la institución.

\section{Calidad Educativa}

Diversos autores han enfatizado en el concepto de calidad educativa, sin embargo, hay que entender que se concibe como algo complejo y que tiene connotaciones históricas y culturales. Carr \& Kemmis (1988) citado Casanova (2012), concibe la calidad de la enseñanza como un proceso de optimización continua de las actividades docentes para apoyar y desarrollar el aprendizaje formativo. Por otra parte, se entiende este concepto como un proceso de Inputs y Outputs, en el que el primero se refiere a los recursos humanos que son los materiales iniciales en los IES Y el segundo se vincula a los logros alcanzados por los estudiantes (Martínez et al., 2016).

Seijas (2002) concibe la calidad educativa en un enfoque económico como una "caja negra" donde el alumno utiliza una serie de recursos cognoscitivos para obtener flujos de salida, resultados o productos. En general, estos productos se definen en términos de los logros cognitivos que los estudiantes han logrado y los resultados obtenidos a través de una serie de pruebas estandarizadas.

Para (Martinzes et al., 2016) la calidad educativa tiene componentes como el contexto en el que se desenvuelve la educación, las autoridades, la propia institución y los docentes. Estos últimos se conciben con calidad cuando son puestos a pruebas su constante preparación, su perfil docente, su ambiente de aprendizaje, su plan anual, su desempeño, planificación de clases, número de alumnos, trabajos colaborativos y asistencia.

\section{Metodología}

El apartado metodológico se planteó el tipo de investigación de acuerdo al Manual de Frascati, como investigación aplicada, donde se parte de una teoría e hipótesis de investigación para ser validadas (Fundación Española de Ciencia y Tecnología, 2002). Debido a su naturaleza, este proyecto se enmarcó en una investigación correlacional- exploratoria; desde el punto de vista correlacional, por cuanto intenta determinar relaciones causales entre las variables objeto de estudio, dado que se pretende establecer la incidencia que tiene el aprendizaje organizacional sobre la calidad educativa de la EFIM. Por otro lado, la investigación se definió exploratoria por la escasa literatura científica sobre la relación entre el aprendizaje organizacional y la calidad educativa. 
Las fuentes primarias de la investigación la conformaron dos encuestas; la primera referida al aprendizaje organizacional, compuesta por dos secciones de preguntas descriptivas abiertas y cerradas. La segunda parte la conformaron preguntas de valoración tipo escala Likert sobre el aprendizaje organizacional al interior de la EFIM. Igualmente, una segunda encuesta relaciona la calidad educativa en la EFIM, vista desde la calidad de los docentes. La población objetivo de la encuesta la constituyeron los estudiantes del primer periodo académico del año 2018 de la EFIM.

Se estableció el nivel de asociación entre el aprendizaje organizacional y la calidad educativa: Para identificar las relaciones entre el aprendizaje organizacional y la calidad educativa en la EFIM, se utilizó el método de ecuaciones estructurales, las cuales permiten contrastar mediante la construcción de un modelo teórico propuesto, la validez de las interrelaciones entre las variables. Se diferencia del análisis factorial exploratorio puesto que el investigador construye con antelación las posibles relaciones causales entre los constructos a someter, empleando como etapas del modelo de ecuaciones estructurales (García-Bravo et al., 2019).

La población objeto del estudio fueron los docentes y administrativos de la EFIM, que dictaron clases para el periodo de 2018-I. Debido a que el universo de profesores y administrativos correspondía a 64 , se decidió aplicar al total de la población y no calcular muestra. No obstante, se realiza una validación del instrumento de calidad educativa con el total de estudiantes que respondieron la encuesta, dado que se midió calidad docente como proxy de calidad educativa (García-Bravo et al., 2019). La encuesta fue aplicada a un total de 478 estudiantes, lo que permitió verificar los factores que determinan la calidad educativa a través de los docentes. Luego, estos resultados fueron normalizados para cada uno de los profesores que impartió las clases en los periodos mencionados y los que fueron objeto de la primera encuesta de aprendizaje organizacional, esto permitió realizar análisis relacional entre el aprendizaje organizacional y la calidad educativa vista desde los docentes que imparten clases en la EFIM.

En cuanto al Instrumento de medición, se utilizaron dos tipos de cuestionario: El primer cuestionario estuvo compuesto por 60 preguntas, discriminadas con variables sociodemográficas (Edad, Grado de Militar y/o civil, Género, Cargo que ocupa, Área, Años de Antigüedad) y variables asociadas al aprendizaje organizacional (Se establecen los constructos e ítems que permitirán medir el aprendizaje organizacional en la EFIM), a través de las dimensiones: Ambiente de soporte para el aprendizaje, Procesos y prácticas concretas de aprendizaje, Liderazgo y refuerzo del aprendizaje basado en Garvin, Edmondson, \& Gino (2008). Las preguntas en esta sección siguieron la lógica de tipo valorativas en escala de 1 a 5 (donde $1=$ totalmente en desacuerdo, $5=$ totalmente de acuerdo).

El segundo cuestionario consistió en medir la calidad educativa vista desde la calidad de sus docentes. No obstante, se procedió a validar el instrumento a través del método de análisis factorial exploratorio. Este instrumento consistía en 19 preguntas de valoración con una escala de 1 a 5 (donde 1 = totalmente en desacuerdo, 5 = totalmente de acuerdo).

\section{Resultados}

Se realizó con expertos inicialmente que permitieran corroborar la construcción sistémica de cada instrumento, a partir de ello recibir recomendaciones de mejora. Una vez verificado el instrumento, se procedió a realizar un test de fiabilidad y consistencia interna a través del estadístico Alfa de Cronbach. Éste plantea ciertas condiciones necesarias para la validación del instrumento: sí es menor a 0,7 , no existe una buena consistencia interna de los ítems. Pero si se encuentra entre los intervalos 0,7-0,9 se considera aceptable y el instrumento presenta una buena consistencia interna (Campo-Arias \& Oviedo, 2008). Los resultados del Alfa de Cronbach se presentan así: 
Tabla 1. Validación de resultados

\begin{tabular}{lcc}
\hline \multicolumn{1}{c}{ Escalas de Medición } & Alpha de Cronbach & No. Ítems \\
\hline Ambiente de soporte para el aprendizaje & $\mathbf{0 , 4 3 4}$ & $\mathbf{1 8}$ \\
Procesos y prácticas concretas de aprendizaje & $\mathbf{0 , 8 9 0}$ & $\mathbf{2 8}$ \\
Liderazgo y refuerzo del aprendizaje & $\mathbf{0 , 7 2 6}$ & $\mathbf{8}$ \\
\hline
\end{tabular}

Fuente: elaboración propia

Dado los resultados del test de fiabilidad de las escalas de aprendizaje organizacional, se determina que la escala de ambiente de soporte para el aprendizaje es menor a 0,7 , por lo cual esta escala no se incluye en los análisis posteriores, puesto que no presenta una buena consistencia interna los ítems, es decir, no está midiendo lo que se requiere medir.
El segundo instrumento de medición, el cual pretende medir la calidad educativa de la EFIM en término de la calidad de sus docentes, no presenta unos factores predeterminados; así las cosas, se establece el alfa de Cronbach para el instrumento en su totalidad. Los resultados se presentan en la siguiente tabla

Tabla 2. Resultados del Alfa de Cronbach

\begin{tabular}{c}
\hline ESTADÍSTICA DE FIABILIDAD \\
\hline Alfa de Cronbach No de elementos \\
0,971 \\
\hline Fuente: elaboración propia
\end{tabular}

Dado que el Alfa de Cronbach del instrumento de calidad educativa se encuentra en 0,971 , se considera que presenta una buena consistencia interna y validez.

Para la identificación se tomó como referencia lo propuesto por Garvin, Edmondson, \& Gino (2008) y a partir de esta propuesta de medición, se realizó un análisis de conglomerados jerárquicos que develo aquellas prácticas referidas al aprendizaje organizacional en la EFIM, clasificándolas en debilidades y fortalezas, así:

- Respuestas Negativas $=\sum$ (Sumatoria): respuestas "En desacuerdo" y "Totalmente en desacuerdo" por parte de los encuestados.

- Respuestas Positivas $=\sum$ (Sumatoria): respuestas "De acuerdo" y "Totalmente de acuerdo" por parte de los encuestados.

- Respuestas neutras $=\sum$ (Sumatoria): respuestas "Ni en desacuerdo ni de acuerdo" por parte de los encuestados.

- La condición para declarar un ítem en debilidad, neutralidad o fortaleza se realizó así:
- Si las respuestas negativas son mayores que las respuestas positivas y las respuestas neutras, entonces existe condición de debilidad del ítem.

- Si las respuestas positivas son mayores que las respuestas negativas y las respuestas neutras, entonces existe condición de fortaleza del ítem.

- Si las respuestas neutras son mayores que las respuestas negativas y las respuestas positivas, entonces existe condición de neutralidad en el ítem.

Los resultados muestran que en el primer conglomerado la dimensión de liderazgo y refuerzo del aprendizaje presenta la mayor fortaleza en la EFIM (100\%), seguido de la dimensión de procesos y prácticas concretas de aprendizaje $(92,85 \%) \mathrm{y}$, por último, la dimensión de menor fortaleza se encuentra la de ambiente para el aprendizaje $(61,11 \%)$. Acorde a la naturaleza de la EFIM, estos resultados son coherentes con las doctrinas practicadas respecto al desarrollo de la organización, siguiendo los lineamientos establecidos por instituciones superiores, pero teniendo autonomía en decisiones meramente organizacionales, del quehacer de la institución (García-Bravo et al., 2019). 
Por parte de las debilidades resultado del análisis de conglomerados de la EFIM, se resalta que la dimensión de ambiente de soporte para el aprendizaje, es la de mayor dificultad con un 38,89\% del total de ítems en debilidades y la dimensión de procesos y prácticas concretas de aprendizaje, obtuvo dos debilidades, teniendo un total de 7,15\%. Estos resultados tienen relación con la manera como está concebida la EFIM, en razón a que, siendo una institución de tipo militar, existen limitaciones sobre todo desde los individuos, en el proceso de compartir conocimiento, el cual constituye una de las bases para el aprendizaje organizacional, sustentando esta debilidad en una limitada autonomía de los individuos con sus superiores, esto incluye tanto el personal civil como el militar.

El desarrollo de esta sección requirió aplicar técnicas estadísticas con el fin de dar cumplimien- to a lo propuesto. Es así que, se realizó un análisis factorial exploratorio que determinara aquellos factores que explican la calidad educativa en la EFIM, basado en una batería de preguntas diseñadas previamente por la Armada Nacional. Este análisis permitió identificar aquellos ítems que no son acordes ni significativos a lo que se pretende medir, por lo tanto, aplicar esta técnica fue de suma importancia para el proyecto de investigación, puesto que una vez identificados los factores que explican la calidad educativa, se procede a medir la influencia de los factores de aprendizaje organizacional en estos.

La aplicación del Análisis Factorial Exploratorio (AFE) requiere la previa validación del test de esfericidad de Bartlett y la prueba de medida de ajuste de Kaiser-Meyer-Olkin KMO, los cuales permiten verificar si es factible la aplicación de la técnica AFE, obteniendo estos resultados:

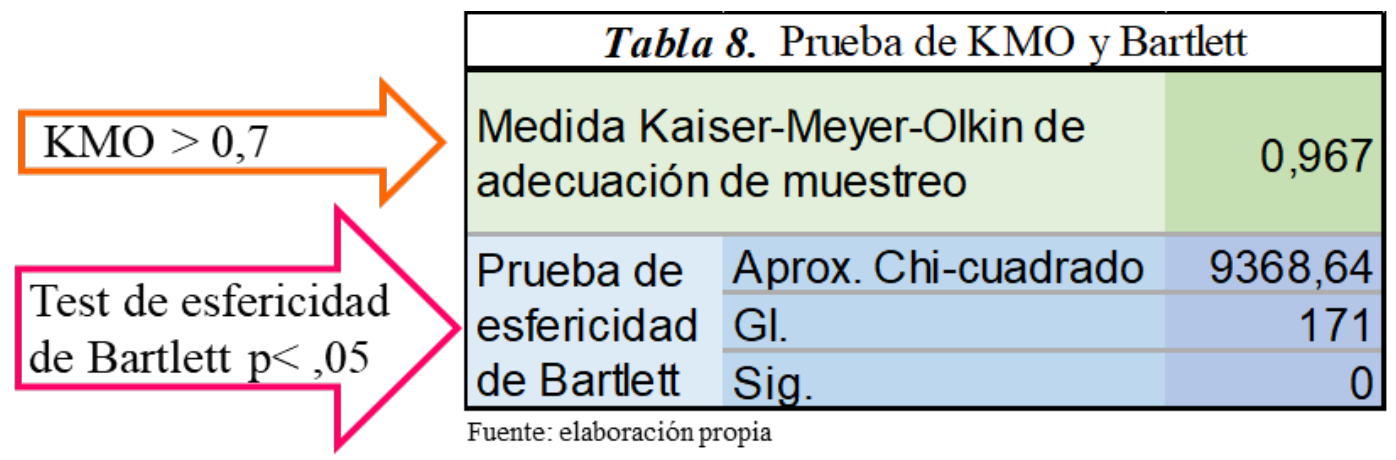

Figura 1. Prueba de KMO y Bartlett

Fuente: elaboración propia

La figura 1 muestra la varianza total explicada una vez extraídos los componentes que exponen la calidad educativa de la EFIM. Esta varianza determina la conformación de dos componentes, los cuales explican en un $73,887 \%$ el total de ítems que conforman el cuestionario. Así las cosas, una primera vista de los resultados se evidencia en la formación de dos componentes explicativos de la calidad educativa.
Para Establecer el nivel de asociación entre el aprendizaje organizacional y la calidad educativa, presentando evidencia estadística sobre estos constructos, fue necesario identificar cuáles son los factores que explican el aprendizaje organizacional en la EFIM, realizando inicialmente la validación de escalas que permitan determinar la significancia estadística de cada una. Para el caso de las no significativas se excluirán de los análisis posteriores.

Tabla 3. Test de fiabilidad - escalas Aprendizaje Organizacional

\begin{tabular}{lcc}
\hline \multicolumn{1}{c}{ Escalas de medición } & Alpha de Cronbach & Cant. Items \\
\hline Ambiente de soporte para el aprendizaje & 0,434 & 18 \\
Procesos y prácticas concretas de aprendizaje & 0,89 & 28 \\
Liderazgo y refuerzo del aprendizaje & 0,726 & 8 \\
\hline
\end{tabular}

Fuente: elaboración propia 
De acuerdo a los resultados del test de fiabilidad de las escalas de aprendizaje organizacional, se determina que la escala de ambiente de soporte para el aprendizaje es menor a 0,7 , motivo por el cual esta escala no se incluye en los análisis posteriores. Una vez validada las escalas, se procede a realizar un análisis factorial exploratorio, que permita reducir las dimensiones e ítems que lo conforman, dando como resultado una nueva configuración, dadas las condiciones específicas en la EFIM. En la siguiente tabla se observa que el valor del KMO es de 0,677 , para este caso, se considera válido puesto que es mayor a 0,6 , condición necesaria para su validez. Por otro lado, respecto al test de esfericidad de Bartlett el cual presentó un valor de, 000 , se considera válido puesto que la condición es que sea menor al p-valor $(0,05)$.

Tabla 4. Prueba de KMO y Bartlett

\begin{tabular}{ccc}
\hline Medida Kaiser-Meyer-Olkin de adecuación de muestreo & 0,677 \\
Prueba de esfericidad de Bartlett & Aprox. Chi-cua- & 1551,312 \\
drado & \\
Gl & 630 \\
& Sig. & 0 \\
\hline
\end{tabular}

Fuente: elaboración propia

Desde estas dos condiciones validadas, es posible aplicar el análisis factorial exploratorio y se procedió a calcular la varianza total explicada determinando la conformación de 10 componentes explicativos del aprendizaje organizacional, los cuales revelan en un $74,93 \%$ el total de ítems que conforman el cuestionario. No obstante, se hizo necesario verificar la conformación de los componentes acorde a lo propuesto por Lloret-Segura, Ferreres-Traver, Hernández-Baeza, \& Tomás-Marco (2014), el cual sugiere que un investigador debe retener el mayor número de componentes en función del número de ítems (mínimo 3-4), tamaño de la saturación (mínimo .40) y significado de los ítems que definen el componente entre otros.

Dado los resultados del análisis factorial exploratorio, la nueva configuración de los factores para el caso de la EFIM, serán los siguientes:

Tabla 5. Cantidad de ítems en cada factor del modelo

\begin{tabular}{lc}
\hline Factores del A.O. y la C.E. para el Modelo & Cant. Ítems \\
\hline $\mathrm{AI}=$ Aprendizaje Individual & 10 \\
$\mathrm{AG}=$ Aprendizaje Organizacional & 6 \\
$\mathrm{EC}=$ Entrenamiento constante & 6 \\
$\mathrm{CC}=$ Captación de conocimiento & 3 \\
$\mathrm{E}=$ Evaluación & 3 \\
$\mathrm{CD}=$ Calidad del Docente & 15 \\
$\mathrm{CP}=$ Calidad del Programa & 4 \\
\hline
\end{tabular}

Fuente: elaboración propia

Luego de obtener los factores para el aprendizaje organizacional y la calidad educativa, el siguiente paso consistió en detallar las características del modelo, basado en la hipóte- sis referente al vinculo existente entre el aprendizaje organizacional y la calidad educativa. 


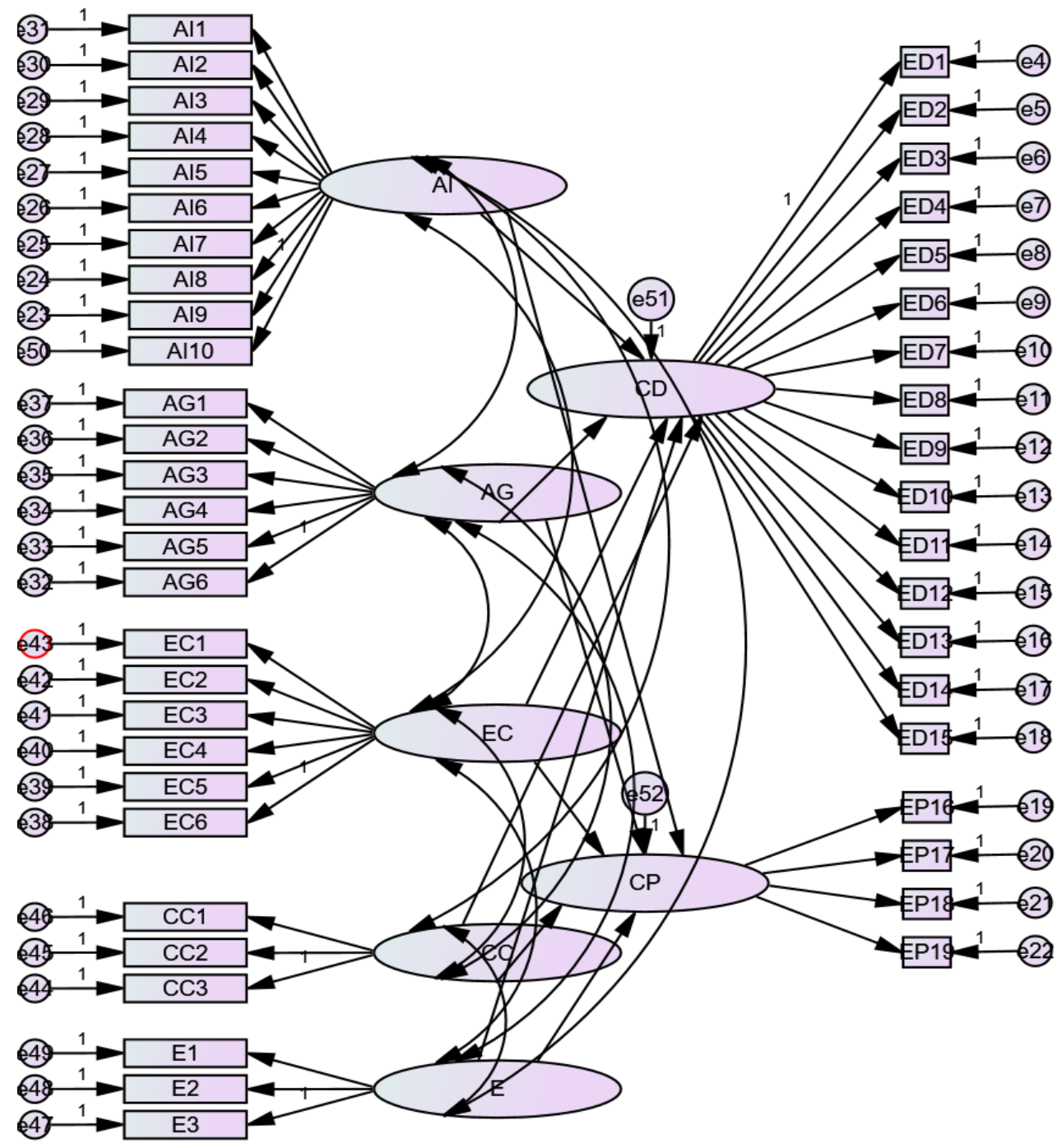

Figura 2. Modelo de Ecuaciones Estructurales

Fuente: elaboración propia

Las variables asociadas a estos, son los ítems que conforman cada factor o dimensión, a su vez estos tienen asociados el error de medida porque cada uno de los factores se comporta como un modelo de regresión.

\section{Conclusiones y Discusiones.}

Los resultados obtenidos avalan, aunque diferencialmente, la hipótesis previa establecida en esta investigación, suponiendo que el aprendizaje organizacional influye sobre la calidad educativa, ya que el Aprendizaje Organizacional permite a las instituciones mejorar los procesos de enseñanza aprendizaje y diseñar estrategias en beneficio de la calidad e incremento de capacidades del ente educativo.
El aprendizaje organizacional como un colectivo no existe sin el aprendizaje individual y solo depende de la apropiación de los conocimientos por parte de las personas que integran el colectivo, el equipo de trabajo o grupo de estudio que generarán competencias distintivas y significativos que perduren y cambien la orientación de la institución educativa (Londoño-Patiño \& Acevedo-Álvarez, 2018).

En complemento al logro de los objetivos planteados, se identificaron los factores que inciden en el aprendizaje organizacional en la EFIM, hallando las fortalezas y debilidades a través de las dimensiones propuestas en los dos instrumentos empleados con los criterios descritos por Garvin, Edmondson, \& 
Gino (2008), indicando que la mayor fortaleza se evidencia en la facilidad que presenta la EFIM para compartir ideas y puntos de vista sobre las labores de los empleados, siempre y cuando no se desvíe de la doctrina castrense. El segundo objetivo específico también se vio cumplido en la medida que se identificaron los factores que inciden en la calidad educativa de la EFIM, empleando una escala de Likert que permitió determinar la conformación de dos componentes explicativos de la Calidad Educativa como propuesta del investigador, (1) Calidad Docente y (2) Calidad del Programa (2), dado las características de los ítems que conformaban el instrumento. El tercer objetivo específico se cumplió parcialmente dado que la ecuación estructural arrojo una matriz no positiva definida.

De esta manera y dado que el modelo de ecuaciones estructurales no pudo ser "corrido" debido al tamaño muestral o a que el modelo no es apropiado a las características de la EFIM, se dispuso un análisis de las correlaciones significativas entre los ítems que hacen parte de la dimensión de calidad educativa y la dimensión de aprendizaje organizacional, para determinar la asociación entre estas variables. A pesar que no determina grado de incidencia, si permite establecer los niveles de asociación entre estos dos constructos, como se muestra a continuación.

Tabla 6. Asociación significativa entre constructos

\begin{tabular}{|c|c|c|}
\hline İtems & & $\begin{array}{c}\text { E1 Se desarrollan frecuentemente Post- } \\
\text { Auditorias y retroalimentaciones de } \\
\text { seguimiento a la acción }\end{array}$ \\
\hline ED11 Considera adecuadas las & Correlación de Pearson &, $426^{* *}$ \\
\hline formas de & Sig. (bilateral) & 0,004 \\
\hline $\begin{array}{l}\text { evaluación utilizadas por el } \\
\text { profesor }\end{array}$ & $\mathrm{N}$ & 45 \\
\hline ED14 Las pruebas de evaluación & Correlación de Pearson & ,399** \\
\hline corresponden a los objetivos del & Sig. (bilateral) & 0,007 \\
\hline curso & $\mathrm{N}$ & 45 \\
\hline
\end{tabular}

Fuente: elaboración propia

Este proceso no obtuvo resultados concluy- concluyendo que para el caso de la Escuela de entes respecto a la influencia del aprendizaje Formación de Infantería de Marina esta condiorganizacional sobre la calidad educativa, a ción no se aplica.

pesar de que existen diversos ítems asociados,

\section{Referencias}

Argyris, C., \& Schon, D. (1999). Organizational learning: a theory of action perspective. California: Addison - Weasly.

Barrientos, J. (2001). Determinantes de la calidad de la educación en Colombia (p. 78). Fedesarrollo.

Bolívar, A. (2001). Los centros educativos como organizaciones que aprenden: una mirada crítica. Contexto Educativo: Revista Digital de Investigación y Nuevas Tecnologías, January 2001, 4 .

Campo-Arias, A., \& Oviedo, H. C. (2008). Propiedades Piscométrica sde una Escala: la Consistencia Interna. Revista de Salud Pública, 10(5), 831-839.

Casanova, M. (2012) El diseño curricular como factor de calidad educativa.Revista Iberoamericana sobre Calidad, Eficacia y Cambio en Educación -Volumen 10, http://www.rinace.net/reice/numeros/arts/ vol10num4/art1.pdf 
Del Río Cortina, J., \& Santisteban, D. (2011). Perspectivas Del Aprendizaje Organizacional Como Catalizador De Escenarios Competitivos Organizational Learning Perspectives As Catalizers of Competitive Scenarios. Revista Ciencias Estratégicas. Revista Ciencias Estratégicas | Medellín -Colombia Revista Ciencias Estratégicas, 19(26), 247-1794.

Fernández, O. (1991). El análisis de cluster: aplicación, interpretación y validación. Papers, 37, 6576.

Fundación Española de Ciencia y Tecnología. (2002). Manual de Frascati - Propuesta de Norma Práctica para Encuestas de Investigación y Desarrollo Experimental. OCDE.

Garbanzo, G. M. (2014). Factores asociados al rendimiento académico en estudiantes universitarios, una reflexión desde la calidad de la educación superior pública. Revista Educación, 31(1), 43. https://doi.org/10.15517/revedu.v31i1.1252

García-Bravo, P., Rodríguez-Arias, C., Martín-León, M., y Silvera-Sarmiento, A. (2019). Global conference on Business and Finance Proceedings. Liderazgo y autoevaluación, para mejorar la gestión directiva educacional, p. 480-491.

Garvin, D., Edmondson, A., \& Gino, F. (2008). Is Yours a Learning Organization? Harvard Business Review, March, 1-9. https://doi.org/10.1037/e413842008-018

Lloret-Segura, S., Ferreres-Traver, A., Hernández-Baeza, A., \& Tomás-Marco, I. (2014). El análisis factorial exploratorio de los ítems : una guía práctica, revisada y actualizada Introducción Determinación de la adecuación del Análisis. Anales De Psicología, 30(3), 1151-1169. https:// doi.org/10.6018/analesps.30.3.199361

Londoño-Patiño, J. A., \& Acevedo-Álvarez, C. A. (2018). El aprendizaje organizacional (AO) y el desempeño empresarial bajo el enfoque de las capacidades dinámicas de aprendizaje. Revista CEA, 4(7), 103-118.

López, P., \& López, J. (2003). ¿Qué es el aprendizaje organizativo? Researchgate, January 2003.

Martínez, G., Guevara, A., \& Valles, M. (2016). El desempeño docente y la calidad educativa. Ra Ximhai, 12(6), 123-134.

Medina, S. (2017). Propuesta de Aplicación del Modelo Integrado de Aprendizaje Organizativo para el Cuerpo Académico del Área de Administración de Empresas de la Facultad de Ciencias Empresariales en la Unipanamericana- Compensar para Promover la Apropiación de una Cultura.

Orozco, J., Olaya, A., \& Villate, V. (2009). ¿ Calidad De La Educación O Educación De Calidad ? Una Preocupación Más Allá Del Mercado. REVISTA IBEROAMERICANA DE EDUCACIÓN, 51, 161-181.

Pérez, E. R., \& Medrano, L. (2010). Análisis Factorial Exploratorio: Bases Conceptuales y Metodológicas. Revista Argentina de Ciencias Del Comportamiento, 2(1), 58-66.

Ramírez-Fernández, R., Machado-Licona, J., \& Fernández-Ramírez, O. (2019). Calidad en la educación universitaria, desde el programa de ingeniería de sistemas: una visión cualitativa de la educación superior. Revista Científica Anfibios, 2(2), 41-50. https://doi.org/10.37979/afb.2019v2n2.49

Schiefelbein, E., \& Schiefelbein, P. (1999). Determinantes de la calidad: ¿qué falta mejorar? 562, 37-64.

Seijas D. A. (2002). Evaluación de la Calidad en Centros Educativos. Coruña, España. 